\title{
The effect of visual angle on global and local reaction times depends on the set of visual angles presented
}

\author{
MARVIN R. LAMB and LYNN C. ROBERTSON \\ Veterans Administration Medical Center, Martinez, California \\ and University of California School of Medicine, Davis, California
}

\begin{abstract}
It has been shown that there is a transition from a global to a local advantage in reaction time as visual angle increases (Kinchla \& Wolfe, 1979), and it has been assumed that this transition reflects lower level (e.g., retinal) processes. In three experiments, we examined whether higher level (e.g., attentional) processes play a role in this transition. In each experiment, subjects received a different stimulus set in each of two blocks of trials. In Experiment 1, stimuli subtending $1.5^{\circ}, 3^{\circ}, 4.5^{\circ}$, or $6^{\circ}$ of visual angle vertically (small-stimuli set) were randomly presented in one block, while the other block consisted of random presentations of $3^{\circ}, 6^{\circ}, 9^{\circ}, 0^{\circ}$ $12^{\circ}$ stimuli (large-stimuli set). The subjects' task was to identify targets that appeared randomly at either the local or the global level. It was found that the transition from a global to a local reaction-time advantage took place at a larger visual angle for the large-stimuli set than for the small-stimuli set. The same effects of stimulus set were found in Experiment 2, in which the smallstimuli set included $1.5^{\circ}, 3^{\circ}$, or $6^{\circ}$ stimuli while the large-stimuli set included $3^{\circ}, 6^{\circ}$, or $9^{\circ}$ stimuli. In Experiment 3, eye position was monitored to rule out the possibility that subjects adopted different fixation strategies depending on which stimulus set was being presented. The findings suggest that attention plays a major role in determining the relative speed of processing of localand global-level information.
\end{abstract}

It has been more than 10 years since Navon (1977) presented his influential series of experiments suggesting that visual pattern processing proceeds from a more global level of structure to a more local level. This global precedence hypothesis was based in part on the fact that reaction times were faster to global (e.g., the large letter in Figure 1) than to local (e.g., the small letters in Figure 1) targets. This difference in reaction time occurred only when the targets appeared within hierarchically organized patterns like those in Figure 1. Performance did not differ for large and small target letters of the same size presented in isolation. This suggested that the difference in reaction times observed by Navon reflected a difference in the order in which the visual system processes different levels of hierarchical structure rather than some lower level sensory effect such as a simple difference in discriminability between smaller and larger targets.

This research was supported by the Medical Research Council of the Veterans Administration, NIH Grant NS27902 to M.R.L., and NIAAA Grant AA06637 to L.C.R. We wish to thank Mirjam Eglin and E. William Yund for their helpful discussions, Linda Kerth for her help with data collection, and John Lackey for computer programming. We also wish to thank Charles W. Eriksen and two anonymous reviewers for their valuable suggestions. Correspondence concerning this article should be addressed to Marvin R. Lamb, Research 151, Veterans Administration Medical Center, 150 Muir Road, Martinez, CA 94553.

\section{$\mathrm{HHHHH}$ H HHHHH H HHHHH}

Figure 1. Drawing of one of the stimuli used in the present study showing a global " $\mathrm{E}$ " composed of local "Hs".

Since Navon reported his findings, a number of investigators have challenged various aspects of the global precedence hypothesis. Several investigators have found a performance advantage for local rather than global targets, suggesting that order of processing is not strictly global to local but can, under the right circumstances, be local to global (Hoffman, 1980; Kinchla \& Wolfe, 1979; Lamb \& Robertson, 1988, 1989; Martin, 1979; Pomerantz \& Sager, 1975). Others, including Navon, have suggested that local and global targets might be processed in parallel or at least with a similar time course (Boer \& Keuss, 1982; Hughes, Layton, Baird, \& Lester, 1984; Miller, 1981; Navon, 1981). The hypothesis that local- and global-level information is processed in parallel is supported by recent neuropsychological evidence that 
suggests that there are two separate mechanisms associated with different cortical regions-one that favors the processing of local information and one that favors the processing of global information (Delis, Robertson, \& Efron, 1986; Lamb, Robertson, \& Knight, 1989, in press; Robertson \& Delis, 1986; Robertson, Lamb, \& Knight, 1988). Thus, there is a great deal of evidence against the notion that local and global information is processed in a fixed order. However, the question remains as to what factors and mechanisms are important in determining the relative speed with which local- and global-level information is analyzed.

There has been a good deal of controversy over whether lower level (e.g., retinal) sensory mechanisms or higher level (e.g., attentional) mechanisms are more important in determining the relative speed of processing of local and global information. Navon's (1977) size control did not answer this question satisfactorily because retinal eccentricity was confounded with hierarchical organization. His single isolated targets were presented foveally, whereas his hierarchically organized patterns were presented peripherally, where reduced acuity would be expected to have a greater detrimental effect on smaller (local) targets. Subsequent studies have provided evidence that retinal location can in fact affect the relative speed with which local and global targets are identified (Grice, Canham, \& Boroughs, 1983; Lamb \& Robertson, 1988; Pomerantz, 1983). This has led to the suggestion that reaction-time differences between local and global targets are due to low-level sensory processes and can be explained simply by differences in discriminability between targets of different sizes.

Kinchla and Wolfe (1979) directly addressed this question by varying the overall visual angle of hierarchically organized patterns. They found a global advantage in reaction time with patterns subtending less than about $7^{\circ}$ of visual angle but a local advantage with larger patterns. The authors suggested that the visual system is organized in such a way that stimuli of a certain fixed size are processed first, and larger or smaller stimuli are processed subsequently.

Although data such as these suggest that low-level sensory processes determine the relative speed with which local and global levels are analyzed, there is also evidence that higher level "top-down" processes play a considerable role. A number of studies have shown that attentional manipulations have a profound effect on relative reaction times to local and global targets (Kinchla, Solis-Macias, \& Hoffman, 1983; Lamb \& Robertson, 1987, 1988; Miller, 1981; Robertson et al., 1988; Ward, 1982). For example, Lamb and Robertson (1988) showed that relative reaction times for foveally presented hierarchical patterns varied depending on whether or not they occurred in the context of peripheral presentations. They argued that when stimuli appeared randomly, either at fixation or in the periphery, subjects were forced to attend to a wider area than when all presentations were central, a distribution of attention that should favor global over local analysis.
This interpretation suggests an alternative to the lowlevel sensory explanation offered for results such as those of Kinchla and Wolfe (1979). Those investigators randomly presented patterns ranging in size from $4.8^{\circ}$ to $22.1^{\circ}$ of visual angle. Presumably, attention would be distributed over the display in such a way as to optimize processing of this particular set of stimuli, and relative performance to local and global targets would reflect this distribution of attention. If so, a set of stimuli with a different range of visual angles should produce a different distribution of attention, thus affecting relative performance to local and global targets. Kinchla and Wolfe found a global advantage for patterns subtending less than about $7^{\circ}$ and a local advantage for larger patterns. If low-level sensory processes are the sole determiner of this effect, then the transition from a global to a local advantage should occur at this same visual angle regardless of the set of stimuli presented to the subject. However, if the visual angle at which this transition occurs varies depending on the visual angles of the stimuli in the set, then it would suggest that higher level (e.g., attentional) processes are involved.

\section{EXPERIMENT 1}

The purpose of Experiment 1 was to determine whether the visual angle at which the transition from a global to a local advantage in reaction time occurs is fixed or varies with context. Subjects identified local and global targets in stimulus patterns that varied randomly in visual angle from trial to trial in a manner similar to that in Kinchla and Wolfe's (1979) experiment. In one block of trials, there were four stimulus patterns ranging from $1.5^{\circ}$ to $6.0^{\circ}$ of visual angle (small-stimuli set). In a separate block of trials, the four patterns ranged between $3^{\circ}$ and $12^{\circ}$ of visual angle (large-stimuli set).

\section{Method}

Subjects. Ten right-handed male volunteers served as subjects. They ranged in age from 48 to 70 years $(M=60.7, S D=7.3)$. (One subject did not return for Day 2 of testing and thus did not participate in the small-stimuli condition.) Visual acuity was measured with a Snellen chart, and all subjects had normal or correctedto-normal acuity. All subjects had performed in earlier experiments with similar stimuli, but Experiment 2 will show that this had little or no effect on the pattern of results observed here. The subjects were paid for their participation.

Apparatus and Stimuli. The stimuli were generated on a Princeton Graphics SR-12 monitor controlled by an 80286 -based microcomputer (IBM AT compatible) with Sigma Designs Graphic Dazzler I and Enhancer cards. The experiment was conducted with ordinary room lighting. All stimulus events were white on a dark surround. The brightness of the letters and the background were approximately 21.9 and $0.7 \mathrm{~cd} / \mathrm{m}^{2}$, respectively, as measured by a Pritchard SPECTRA photometer. Stimulus timing (onset, offset, and duration) was tied to the vertical sync pulse (refresh rate approximately $57 \mathrm{~Hz}$ ). All other events [responses, intertrial interval (ITI), etc.] were timed using the 8253 chip set to a $1-\mathrm{msec}$ time base. The status of the response keys was monitored via the game port.

A set of stimulus patterns formed from the letters " $\mathrm{H}$," " $\mathrm{S}$," "A," and "E"' were used (see Figure 1). Global letters were constructed from the appropriate placement of local letters in a $5 \times 5$ 
matrix. The vertical aspect of the global pattern subtended either $1.5^{\circ}, 3^{\circ}, 4.5^{\circ}$, or $6^{\circ}$ (small-stimuli set), or $3^{\circ}, 6^{\circ}, 9^{\circ}$, or $12^{\circ}$ (largestimuli set). Global letters were 7.4 times as tall as local letters. Both global and local letters were 1.5 times as tall as they were wide. The line segments that made up the stimulus patterns were approximately $1 \mathrm{~mm}$ thick.

The letters " $H$ " and " $S$ " served as targets, and the letters " $A$ " and " $E$ " served as distractors. All possible combinations of these letters appeared equally often, with the restriction that each stimulus contain one target and one distractor letter. All stimulus events occurred in the center of the screen.

Procedure. The subject sat with his head resting against the back of a large easy chair. The distance between the subject's eyes and the CRT screen was approximately $71 \mathrm{~cm}$. A $500-\mathrm{msec}$ tone announced the beginning of each trial. The tone was followed by a 100 -msec pause and then by a $100-\mathrm{msec}$ presentation of one of the stimulus patterns. The subject's task was to indicate, by pressing one of two response keys, which of the target letters (" $\mathrm{H}$ " or " $\mathrm{S}$ ") was present on that trial. A 1-sec ITI occurred following a response or after $3 \mathrm{sec}$ if no response occurred.

There were four blocks of 67 trials on each of 2 days of testing. The first three trials of each block were warm-up trials that were not included in the analysis. On Day 1 each block contained an equal number of each stimulus in the large-stimuli set, and on Day 2 each block contained an equal number of each stimulus in the smallstimuli set. (Stimulus set was not counterbalanced because data from Day 1 were being collected for comparison with brain-injured subjects in another experiment. Although confounding stimulus set with set order could affect the interpretation of the data, the results of Experiment 2 show that order of presentation does not affect the pattern of results.) Before data collection began each day, the subjects received a block of 64 practice trials. Stimuli were presented randomly, with the restrictions that target letter, distractor letter, target level, and visual angle were completely counterbalanced within each block, and that the same visual angle could not occur on more than three consecutive trials.

Each subject used his right hand to respond, and pressed the " $H$ " key with the index finger and the " $S$ " key with the middle finger. The subjects were instructed to respond as quickly as possible while keeping errors to a minimum. Reaction time (measured from stimulus-pattern onset to key closure) and errors were recorded.

\section{Results}

Error rates were low for both the large-stimuli set $(M=1.3 \%)$ and the small-stimuli set $(M=3.2 \%)$. A separate repeated measures analysis of variance (ANOVA) was performed on the reaction-time data for each stimulus set. The factors included were visual angle $\left(3^{\circ}, 6^{\circ}\right.$, $9^{\circ}$, or $12^{\circ}$ for the large-stimuli set, and $1.5^{\circ}, 3^{\circ}, 4.5^{\circ}$, or $6^{\circ}$ for the small-stimuli set) and level (local or global, for both sets). Median reaction times were calculated for each cell in the design, and the data reported in the figures and in the ANOVAs are means of those medians.

Small-stimuli set. The reaction-time data are presented in Figure 2. Overall, reaction times for the small-stimuli set decreased as the visual angle of the pattern increased $[F(3,24)=47.36, p<.001]$. More importantly, the relative reaction times to local and global targets varied with visual angle, as evidenced by a significant visual angle $\times$ level interaction $[F(3,24)=40.89, p<.001]$. The source of this interaction is important because it reflects the transition from a global advantage in reaction time to a local advantage. Inspection of Figure 2 shows that

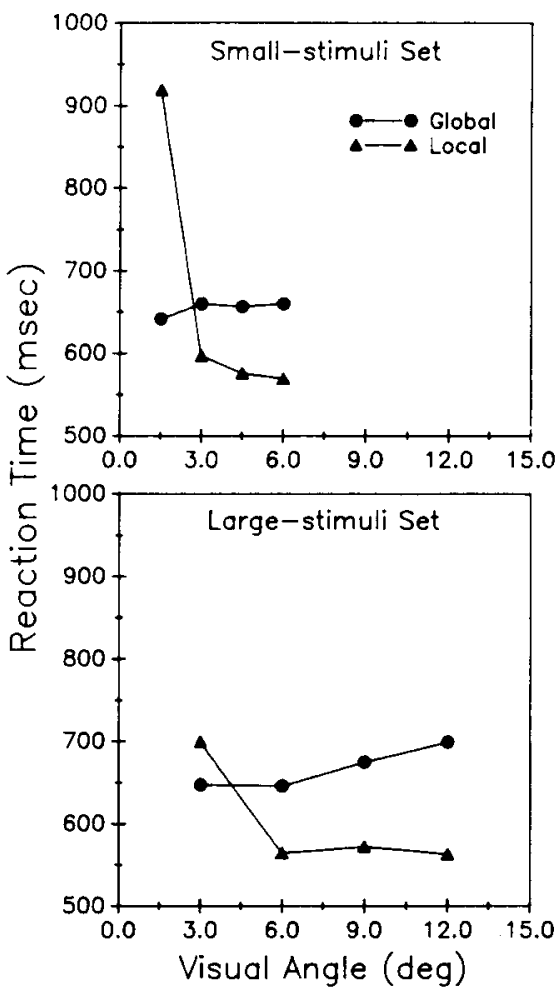

Figure 2. Reaction times for Experiment 1 as a function of visual angle for local (triangles) and global (circles) targets. The top panel shows the data for the small-stimuli set and the bottom panel shows the data for the large-stimuli set.

this transition occurred between $1.5^{\circ}$ and $3^{\circ}$ for the smallstimuli set, and an analysis that included just these two visual angles produced a significant visual angle $\times$ level interaction $[F(1,9)=36.68, p<.001]$. In contrast, there was no visual angle $\times$ level interaction when the three larger patterns of the small-stimuli set were compared. Instead, there was a local advantage in reaction time $[F(1,9)=22.75, p<.001]$ that did not change as a function of visual angle. This local advantage was reversed to a global advantage for the $1.5^{\circ}$ patterns $[F(1,8)=47.53$, $p<.001]$.

Large-stimuli set. Overall, the subjects responded faster to local than to global targets when presented with the large-stimuli set $[F(1,9)=7.39, p<.05]$, and reaction times varied as a function of the visual angle of the pattern $[F(3,27)=7.63, p<.001]$. More importantly, as with the small-stimuli set, there was a significant interaction between visual angle and level $[F(3,27)=$ $8.69, p<.001]$, reflecting a transition from a global to a local advantage in reaction time. Although this transition occurred between $1.5^{\circ}$ and $3^{\circ}$ for the small-stimuli set, it occurred between $3^{\circ}$ and $6^{\circ}$ for the large-stimuli set. There was a visual angle $\times$ level interaction when the $3^{\circ}$ and $6^{\circ}$ patterns were considered alone $[F(1,9)=$ $6.44, p<.05$ ], and although this interaction also reached significance for the three larger patterns $[F(2,18)=3.55$, 
$p<.05$ ], the local advantage associated with the larger patterns was still clearly present at $6^{\circ}[F(1,9)=15.26$, $p<.01]$. Although there was a mean global advantage at $3^{\circ}$, this difference did not reach statistical significance.

\section{Discussion}

The present data are consistent with those of Kinchla and Wolfe (1979) in that there was a mean global advantage in reaction time for smaller patterns but a local advantage in reaction time for larger patterns. However, the transition from a global to a local advantage occurred at different visual angles for the two stimulus sets used here and at yet a third point in Kinchla and Wolfe's experiment. The transition occurred between $1.5^{\circ}$ and $3^{\circ}$ for our small-stimuli set, which ranged in size from $1.5^{\circ}$ to $6^{\circ}$. The transition occurred between $3^{\circ}$ and $6^{\circ}$ for our large-stimuli set, which ranged in size from $3^{\circ}$ to $12^{\circ}$. Finally, the transition occurred between $6.7^{\circ}$ and $10.3^{\circ}$ for Kinchla and Wolfe's set, which ranged in size from $4.8^{\circ}$ to $22.1^{\circ}$. Thus, the visual angle at which the transition occurs depends on the set of visual angles presented to the subject: the larger the stimuli in the set, the greater the visual angle at which the transition will occur.

These data are not consistent with Kinchla and Wolfe's (1979) suggestion that the visual system favors the processing of stimuli of a specific fixed size. What constitutes the optimal size depends on the context-in this case, on the set of visual angles experienced by the subject. Furthermore, these data are not consistent with the idea that the relative speed with which local and global levels are processed is determined simply by low-level sensory processes (e.g. , the retinal mosaic) that might affect the relative discriminability of the two levels (Grice et al., 1983; Hoffman, 1980; Kinchla \& Wolfe, 1979; Pomerantz, 1983).

\section{EXPERIMENT 2}

Experiment 2 was designed to examine some of the factors that may have influenced the point at which the transition from a global to a local advantage occurred in Experiment 1. As in Experiment 1, subjects received two stimulus sets in separate blocks of trials, and both sets had identical $3^{\circ}$ and $6^{\circ}$ patterns. Unlike Experiment 1, however, it was not the case that one set had an intermediate (i.e., $4.5^{\circ}$ ) visual angle and one set did not. Instead, the two sets differed only in that the large-stimuli set contained one larger pattern $\left(9^{\circ}\right)$ and the small-stimuli set contained one smaller pattern $\left(1.5^{\circ}\right)$. In addition, stricter control over the visual angle of the stimuli was obtained through the use of a head restraint, which ensured that the distance between the screen and the subject was fixed. Finally, the order of presentation of the two sets was counterbalanced to control for possible practice effects.

\section{Method}

Subjects. Ten right-handed male volunteers recruited from a local college served as subjects. None had been involved in previous experiments using procedures or stimuli similar to those used here. They ranged in age from 18 to 31 years $(M=23.2, S D=4.2)$. All subjects had normal or corrected-to-normal vision as tested by Snellen chart. The subjects were paid for their participation.

Apparatus and Stimuli. All aspects of the apparatus were the same as in Experiment 1, except that the distance between the subject's eyes and the CRT screen was fixed with the use of an Applied Science Laboratories Model 115 chinrest and head restraint. Like Experiment 1, Experiment 2 was conducted with ordinary room lighting. The two experiments were conducted in different rooms, so the luminance values differed in the two experiments. In Experiment 2, the brightness of the letters and the background were approximately 26.0 and $5.8 \mathrm{~cd} / \mathrm{m}^{2}$, respectively. The visual angles used in Experiment 2 were a subset of those used in Experiment 1 . The vertical dimension of the global pattern subtended either $1.5^{\circ}, 3^{\circ}$, or $6^{\circ}$ (small-stimuli set), or $3^{\circ}, 6^{\circ}$, or $9^{\circ}$ (large-stimuli set).

Procedure. The procedure used in Experiment 2 was the same as that used in Experiment 1, except that (1) all testing occurred on a single day for each subject, (2) the order in which the two stimulus sets were presented was counterbalanced among subjects, and (3) the number of trials was reduced due to the smaller set sizes. Half of the subjects received the large-stimuli set ( 96 trials) followed by the small-stimuli set ( 96 trials), and the rest received the two sets in the reverse order. The subjects received a block of 16 practice trials before the beginning of data collection for each stimulus set.

\section{Results and Discussion}

Error rates were low for both the large-stimuli $(M=$ $4.6 \%)$ and the small-stimuli $(M=5.5 \%)$ sets. A separate repeated measures ANOVA was performed on the reaction-time data for each stimulus set. The factors included were visual angle $\left(1.5^{\circ}, 3^{\circ}\right.$, or $6^{\circ}$ for the smallstimuli set, and $3^{\circ}, 6^{\circ}$, or $9^{\circ}$ for the large-stimuli set), level (local or global, for both sets), and block (first or second, for both sets).

There was no evidence that the order in which the different sets of stimuli were presented influenced the subjects' performance. There was no main effect of block, and block did not interact with any other factor for either the large-stimuli or the small-stimuli set.

As in Experiment 1, there was an interaction between visual angle and level for both the small-stimuli set $[F(2,16)=8.46, p<.01]$ and the large-stimuli set $[F(2,16)=8.37, p<.01]$ (see Figure 3). More importantly, just as in Experiment 1, the source of this interaction differed for the two stimulus sets, indicating that the transition from a global advantage to a local advantage in reaction time occurred at different visual angles for the two sets of stimuli. For the small-stimuli set, the visual angle $\times$ level interaction occurred between $1.5^{\circ}$ and $3^{\circ}[F(1,9)=12.28, p<.01]$, and there was no such interaction between $3^{\circ}$ and $6^{\circ}$. In contrast, for the largestimuli set, there was a visual angle $\times$ level interaction between $3^{\circ}$ and $6^{\circ}[F(1,9)=8.7, p<.05]$, but there was no such interaction between $6^{\circ}$ and $9^{\circ}$.

The influence of set on relative global/local advantage is clearly demonstrated by the difference in performance between the two stimulus sets at $3^{\circ}$ and $6^{\circ}$-the visual angles shared by the two sets. The $3^{\circ}$ and $6^{\circ}$ patterns were identical in the two sets, yet there was a crossover inter- 


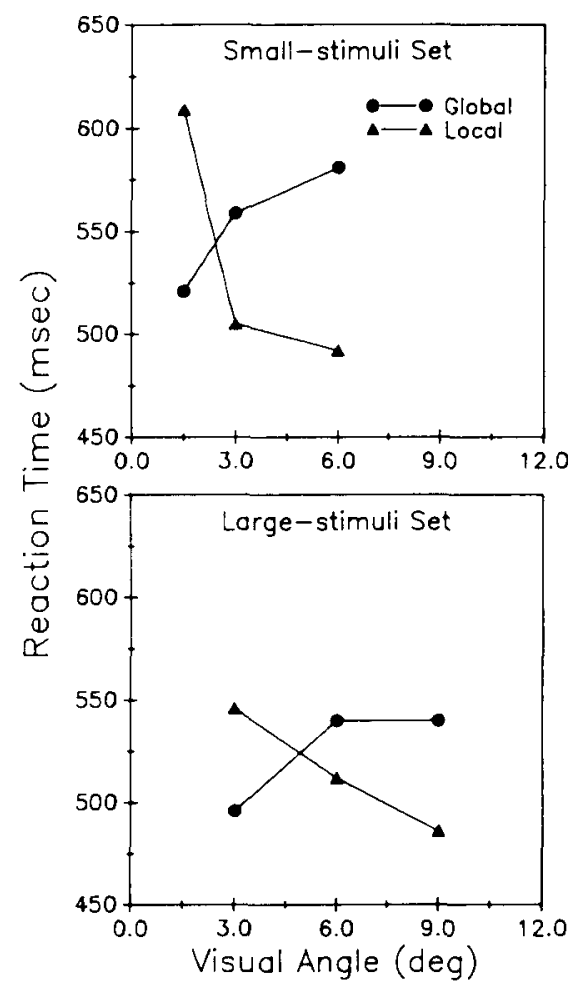

Figure 3. Reaction times for Experiment 2 as a function of visual angle for local (triangles) and global (circles) targets. The top panel shows the data for the small-stimuli set and the bottom panel shows the data for the large-stimuli set.

action between these two visual angles for the large-stimuli set but not for the small-stimuli set.

The results of Experiment 2 replicated those of Experiment 1 . The transition point from a global to a local advantage changed as a function of stimulus set even though the large-stimuli and small-stimuli sets differed by only a single value in Experiment 2. In addition, the results of Experiment 2 showed that the change in the transition point was unaffected by the order in which the sets were experienced or by the presence of intermediate visual angles. Finally, the transition point changed in the same way regardless of whether the two stimulus sets were presented in close temporal succession (Experiment 2) or several days apart (Experiment 1). These results show that the process responsible for the change in the transition point is sensitive to small differences between stimulus sets and is a dynamic process that can act over a relatively short time period.

\section{EXPERIMENT 3}

In Experiments 1 and 2, the visual angle at which the transition from a global to a local advantage in reaction time occurred differed depending on the set of visual angles employed. These results suggest that the effect of visual angle on relative level advantage is not due solely to low-level sensory processes affecting the relative discriminability of local and global targets. If it were, the relative response times to local and global targets should have been the same for patterns of a given size, regardless of the set of stimuli within which those patterns occurred. However, this conclusion rests on the assumption that the subjects did not adopt different fixation strategies depending on which stimulus set was presented. That is, it assumes that patterns of a given size fell on the same retinal locus regardless of the set of stimuli presented. Since eye position was not monitored in Experiments 1 and 2 , it is possible that the subjects adopted different fixation strategies for the different stimulus sets. For example, assume the subjects always fixated the location where the outside edge of the largest pattern in the set falls. This would mean that the local targets of a $6^{\circ}$ pattern would be foveated if that were the largest pattern in the set, but would not be foveated if the set contained a $9^{\circ}$ pattern. It is clear that such a fixation strategy could change the discriminability of, and thus the reaction times to, the local targets of identical $6^{\circ}$ patterns, depending on which set they were in.

The purpose of Experiment 3 was to determine whether or not the effects observed in the first two experiments could be due to the use of different fixation strategies for the different stimulus sets. Experiment 3 repeated the procedure used in Experiment 2, except that on each trial a fixation point preceded the presentation of the hierarchical pattern. The subjects were instructed to keep their eyes on the fixation point, and an eye-movement monitor was used to determine eye position. A hierarchical stimulus was not presented until it was determined, by the use of the eye-movement monitor, that the subject was looking at the fixation point. The stimulus was then presented for $100 \mathrm{msec}$, as in Experiments 1 and 2. This procedure ensured that the subjects fixated approximately the same point on all trials for both stimulus sets.

\section{Method}

Subjects. Ten right-handed male volunteers recruited from a local college served as subjects. None had been involved in previous experiments using procedures or stimuli similar to those used here. They ranged in age from 18 to 30 years $(M=22.8, S D=4.2)$. All subjects had normal vision and were paid for their participation.

Apparatus, Stimuli, and Procedure. The apparatus, stimuli, and procedure used in Experiment 3 were identical to those used in Experiment 2, with the following exceptions. Eye movements were monitored using an Applied Science Laboratories Model 210 eyemovement monitor. The monitor was interfaced with the computer controlling the experimental events, and information about eye position was used to determine trial events. The sequence of trial events was the same as in Experiment 2, except that a central fixation point (a filled square subtending approximately $0.16^{\circ}$ of visual angle) was presented $100 \mathrm{msec}$ after offset of the warning tone. Eye position was sampled $400 \mathrm{msec}$ after the onset of the fixation point and every 100 msec thereafter. The fixation point stayed on until eye position was within $0.5^{\circ}$ of the fixation point for two consecutive samples. The hierarchical stimulus appeared $17 \mathrm{msec}$ (one screen refresh) after fixation offset.

\section{Results and Discussion}

Error rates were low for both the small-stimuli ( $M=$ $3.3 \%)$ and the large-stimuli $(M=2.1 \%)$ sets. A sep- 
arate repeated measures ANOVA was performed on the reaction-time data for each stimulus set. The factors included were visual angle $\left(1.5^{\circ}, 3^{\circ}\right.$, or $6^{\circ}$ for the smallstimuli set, and $3^{\circ}, 6^{\circ}$, or $9^{\circ}$ for the large-stimuli set) and level (local or global, for both sets).

As in Experiment 2, there was an interaction between visual angle and level for both the small-stimuli set $[F(2,18)=10.16, p<.001]$ and the large-stimuli set $[F(2,18)=14.89, p<.001]$ (see Figure 4). More importantly, just as in Experiment 2, the source of this interaction differed for the two stimulus sets. For the smallstimuli set, the visual angle $\times$ level interaction occurred between $1.5^{\circ}$ and $3^{\circ}[F(1,9)=11.19, p<.01]$, and there was no such interaction between $3^{\circ}$ and $6^{\circ}$. This replicates the findings of Experiment 2 for the smallstimuli set. For the large-stimuli set, the visual angle $X$ level interaction occurred between $6^{\circ}$ and $9^{\circ}[F(1,9)=$ $31.76, p<.001]$, and there was no such interaction between $3^{\circ}$ and $6^{\circ}$. This replicates the findings of Experiment 2 in that the transition point from a global to a local advantage occurred between larger visual angles for the large-stimuli set than was the case for the small-stimuli set. However, although the transition occurred between $3^{\circ}$ and $6^{\circ}$ for the large-stimuli set in Experiment 2, it did not occur until after $6^{\circ}$ in Experiment 3.

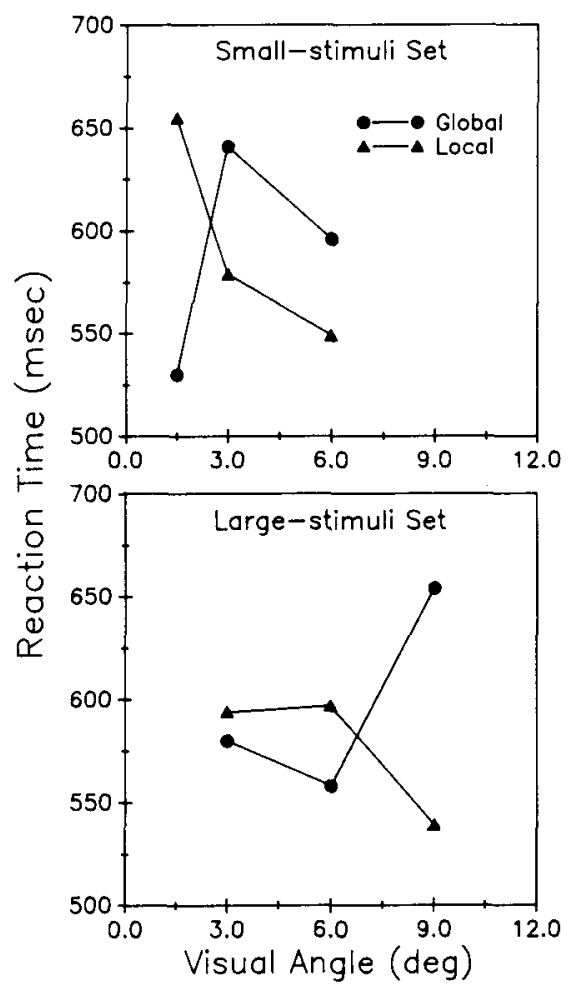

Figure 4. Reaction times for Experiment 3 as a function of visual angle for local (triangles) and global (circles) targets. The top panel shows the data for the small-stimuli set and the bottom panel shows the data for the large-stimuli set.
Although the results of Experiments 2 and 3 were the same in the most important respects, there were differences in the data from the two experiments. As already noted, the transition occurred between $3^{\circ}$ and $6^{\circ}$ for the large-stimuli set in Experiment 2, but between $6^{\circ}$ and $9^{\circ}$ in Experiment 3. In addition, reaction times tended to be longer and more variable in Experiment 3 than in Experiment 2 . The reason for these discrepancies is not clear. The procedures in the two experiments were identical except for the addition of the fixation routine and the monitoring of eye movements in Experiment 3. One possibility is that eye position varied more in Experiment 2 than in Experiment 3, and that this accounts for the differences in performance between the two experiments. Another possibility is that the process of monitoring eye movements itself affected performance. The subjects in Experiment 3 wore glasses with sensors mounted on the frames that were clearly in the field of view, and this might have affected performance. Furthermore, since the presentation of the hierarchical stimulus depended on the subjects' proper fixation in Experiment 3, the timing of its onset was more variable than in Experiment 2, where it always occurred exactly $100 \mathrm{msec}$ after the warning tone. In either case, the differences in performance between Experiments $\mathbf{2}$ and $\mathbf{3}$ do not affect the conclusion of importance here: that the effect of visual angle on relative level advantage depends on the set of visual angles presented. The important finding is that, just as in Experiments 1 and 2, the transition from a global to a local advantage in reaction time occurred at larger visual angles for the set that contained larger patterns. Furthermore, the results of Experiment 3 show that this effect does not depend on the use of different fixation strategies for the different sets.

\section{GENERAL DISCUSSION}

The present data argue against the idea that the relative speed of processing of local and global information is solely dependent on low-level sensory processes that determine the relative discriminability of local and global targets. It is not the case that local targets are identified more slowly than global targets for patterns of a given size simply because limited acuity makes the smaller local targets less discriminable. If this were the case, the relative speed with which local and global targets are identified should be fixed for any given size of pattern. Whatever acuity problem might exist for patterns of a given size should remain, regardless of whether or not patterns of other sizes are also presented. Yet the absolute size of the pattern did not determine relative local and global reaction times in the present experiments. Instead, relative reaction times depended on which visual angles were included in the stimulus set.

The present data are consistent with the hypothesis that attention is an important determiner of the relative speed of processing of local and global information (Kinchla 
et al., 1983; Lamb \& Robertson, 1987, 1988; Miller, 1981; Robertson et al., 1988; Ward, 1982). For example, it has been argued that one mechanism affecting relative level advantage is the size of the attended area. According to this hypothesis, local processing is facilitated when attention is focused on a small area, whereas global processing is facilitated by a broader focus of attention (Lamb \& Robertson, 1988; Ward, 1982). Lamb and Robertson (1988) showed that reaction times to the local level of foveally presented stimuli were slower if those stimuli occurred randomly and were intermixed with peripherally presented stimuli than if all stimuli occurred in the center. They argued that this change in local reaction times reflected the benefit derived from maintaining a smaller focus of attention when all stimuli were presented centrally. A similar account can be offered for the present results (and for those of Kinchla \& Wolfe, 1979). Attention should have been distributed over a wider area for the large-stimuli set than for the small-stimuli set to accommodate the larger stimuli in that set. This increase in the size of the attended area should in turn benefit global over local processing.

Another possibility is that the different stimulus sets affected the distribution of attention among spatial frequency channels rather than the distribution of attention in space. There is recent evidence suggesting that different spatial frequency channels may be involved in processing localand global-level information and that there is selective attention among these channels (Shulman, Sullivan, Gish, \& Sakoda, 1986; Shulman \& Wilson, 1987). Shulman et al. (1986) adapted subjects to sign-wave gratings of different spatial frequencies and then had them identify target letters at either the local or the global level. They found that the adapting frequency that most affected performance on the global task was lower than the adapting frequency that most affected performance on the local task, which suggests that higher spatial frequency channels contribute more to identifying local-level information and lower spatial frequency channels contribute more to identifying global-level information. Furthermore, Shulman and Wilson (1987) provided evidence suggesting that the changes in reaction time to local and global targets caused by attentional manipulations result from selection among these different spatial frequency channels. In their experiment, subjects were told to identify letters at either the local or the global level in separate blocks of trials, in an attempt to induce the subjects to attend selectively. On a small number of probe trials, the subjects were also asked to detect sign-wave gratings of different spatial frequencies. Low-frequency gratings were detected more easily than high-frequency gratings in the context of the global task, whereas the reverse was true in the context of the local task. In the present experiments, it could be that the subjects attended to lower spatial frequencies for the large-stimuli set than for the small-stimuli set in order to accommodate the lower spatial frequencies of the larger stimuli in that set, thus facilitating global processing relative to local processing.
The present data cannot distinguish between these two attentional hypotheses. In our opinion, there is good evidence that both mechanisms operate. Some data are not easily accounted for by the spatial frequency hypothesis (Lamb \& Robertson, 1988), and others cannot be accounted for by the spatial attention hypothesis (Paquet $\&$ Merikle, 1988). It seems quite possible that both mechanisms may have been operating in the present experiments. In any case, the important point here is that the present data show that higher level, top-down processes are important in determining the relative speed with which local and global information is processed.

\section{REFERENCES}

Boer, L. C., \& Keuss, P. J. G. (1982). Global precedence as a postperceptual effect: An analysis of speed-accuracy tradeoff functions. Perception \& Psychophysics, 31, 358-366.

Delis, D. C., Robertson, L. C., \& Efron, R. (1986). Hemispheric specialization of memory for visual hierarchical stimuli. Neuropsychologia, 24, 205-214.

Grice, G. C., Canham, L., \& Boroughs, J. M. (1983). Forest before trees? It depends where you look. Perception \& Psychophysics, 33, 121-128.

HoFFMAN, J. (1980). Interaction between global and local levels of form. Journal of Experimental Psychology: Human Perception \& Performance, 6, 222-234.

Hughes, H. C., Layton, W. M., Baird, J. C., \& Lester, L. S. (1984). Global precedence in visual pattem recognition. Perception \& Psychophysics, 35, 361-371.

Kinchla, R. A., Solis-Macias, V., \& Hoffman, J. (1983). Attending to different levels of structure in a visual image. Perception \& Psychophysics, 33, 1-10.

Kinchla, R. A., Wolfe, J. M. (1979). The order of visual processing: "Top down," "Bottom up," or "Middle out." Perception \& Psychophysics, 25, 225-231.

LAmb, M. R., Robertson, L. C. (1987). The effects of acute alcohol on attention and the processing of hierarchical patterns. Alcoholism: Clinical \& Experimental Research, 11, 243-248.

LAmb, M. R., Robertson, L. C. (1988). The processing of hierarchical stimuli: Effects of retinal locus, locational uncertainty, and stimulus identity. Perception \& Psychophysics, 44, 172-181.

LAMB, M. R. , \& RobertSON, L. C. (1989). Do response time advantage and interference reflect the order of processing of global- and local-level information? Perception \& Psychophysics, 46, 254-258.

Lamb, M. R., Robertson, L. C., KNight, R. T. (1989). Attention and interference in the processing of hierarchical pattems: Inferences from patients with right and left temporal-parietal lesions. Neuropsychologia, 27, 471-483.

LAMb, M. R., Robertson, L. C., \& KNiGht, R. T. (in press). Component mechanisms underlying the processing of hierarchically organized patterns: Inferences from patients with unilateral cortical lesions. Journal of Experimental Psychology: Learning, Memory, \& Cognition.

MARTIN, M. (1979). Local and global processing: The role of sparsity. Memory \& Cognition, 7, 476-484.

Miller, J. (1981). Global precedence in attention and decision. Journal of Experimental Psychology: Human Perception \& Performance, 7, 1161-1174.

Navon, D. (1977). Forest before trees: The precedence of global features in visual perception. Cognitive Psychology, 9, 353-383.

Navon, D. (1981). Do attention and decision follow perception? Comment on Miller. Journal of Experimental Psychology: Human Perception \& Performance, 7, 1175-1182.

Paquet, L., \& Merikle, P. M. (1988). Global precedence in attended and nonattended objects. Joumal of Experimental Psychology: Human Perception \& Performance, 14, 89-100.

Pomerantz, J. R. (1983). Global and local precedence: Selective at- 
tention in form and motion perception. Journal of Experimental Psychology: General, 112, 516-540.

Pomerantz, J. R., \& SAGER, L. C. (1975). Asymmetric integrality with dimensions of visual pattern. Perception \& Psychophysics, 18, 460-466.

RoBertson, L. C., \& Delis, D. C. (1986). "Part-whole" processing in unilateral brain damaged patients: Dysfunction of hierarchical organization. Neuropsychologia, 24, 363-370.

Robertson, L. C., LAMb, M. R., \& KNight, R. T. (1988). Effects of lesions of temporal-parietal junction on perceptual and attentional processing in humans. Journal of Neuroscience, 8, 3757-3769.
Shulman, G. L., Suluivan, M. A., Gish, K., \& Sakoda, W. J. (1986). The role of spatial-frequency channels in the perception of local and global structure. Perception, 15, 259-273.

Shulman, G. L., \& WiLSON, J. (1987). Spatial frequency and selective attention to local and global information. Perception, 16, 89-101.

WARD, L. M. (1982). Determinants of attention to local and global features of visual forms. Journal of Experimental Psychology: Human Perception \& Performance, 8, 562-581.

(Manuscript received October 24, 1988; revision accepted for publication December $1,1989$. ) 